\title{
Extrarenal Angiomyolipoma of Paratesticular and Subcutaneous Region: A Report of Two Cases at Rare Sites
}

\author{
B Rajeshwari*, Shanmugam Salapathi and Mitra Ghosh
}

Department of Histopathology, Apollo Speciality Hospitals, Vanagaram, Chennai -600095. India

\begin{abstract}
Angiomyolipomas are benign mesenchymal neoplasms consisting of variable proportion of mature adipose tissue, thick walled blood vessels and smooth muscle bundles. Although initially thought to be hamartomas, now they are considered to be tumours derived from perivascular epithelioid cells(PEC) hence grouped under a family of tumours known as PEComas. The tumour mainly involves kidneys and are more common in patients with tuberous sclerosis. However extrerenal angiomyolipomas are being increasingly described with liver and retroperitoneum being the most common extrarenal sites. Other sites described include nasal cavity, oral cavity, colon, lung, skin, adrenal glands and bladder. Here we report two cases of extrarenal angiomyolipomas of paratesticular and subcutaneous region, the rarely described sites in the literature. There were no clinical signs of tuberous sclerosis in both cases. Although literature suggests that these tumours show immunohistochemical coexpression of myogenic and melanocytic markers with consistent expression of HMB-45, our both cases were negative for HMB-45. However both cases showed classical triphasic morphology. Although rare, it is important to screen these patients for tuberous sclerosis associated lesions for early diagnosis.
\end{abstract}

Keywords: Angiomyolipoma, Epididymo-orchitis, Extrarenal angiomyolipoma, PEComa, Tuberous sclerosis, HMB-45

\section{Introduction}

Angiomyolipoma(AML) is a benign mesenchymal tumour composed of variable proportion of blood vessels, adipose tissue and smooth muscle. It was initially considered to be a hamartoma, now it is considered to be a tumour derived from perivascular epithelioid cells (PEC), hence grouped under a family of perivascular epithelioid cell tumours known as PEComas. ${ }^{[1]}$ Majority of the cases are sporadic, $20 \%$ of cases are known to occur in association with tuberous sclerosis. ${ }^{[2]}$ However $75 \%$ of patients with Tuberous sclerosis complex(TSC) are associated with renal AMLs. ${ }^{[3]}$ Although it mainly involves the kidneys, extrarenal locations are being increasingly described. Extrarenal Angiomyolipoma (ERAMLs) is usually not associated with tuberous sclerosis. ${ }^{[4]}$ Common extrarenal sites include liver and retroperitoneum. AML of paratesticular and subcutaneous region is extremely rare and very few cases are reported. Here we report two cases of extrarenal angiomyolipoma of paratesticular and subcutaneous location, the rarely described sites in the literature.

\section{Case Reports}

Case 1: A 66 yr old gentleman presented with pain and swelling in right scrotal region since 15 days. Pain was gradually increasing and throbbing. History of on and off fever was present since 15 days. On examination, right scrotum was enlarged and tender. Clinical diagnosis of pyocele was made. On general examination, no clinical signs of tuberous sclerosis were seen. Systemic examination did not reveal any other significant clinical findings. Scrotal ultrasound showed features of pyocele. Ultrasound of abdomen and pelvis was within normal limits. Patient underwent right orchidectomy. Grossly testis with epididymis measured $5 \times 3 \times 3 \mathrm{~cm}$. Testis was grossly unremarkable. The paratesticular region was expanded by a well defined grey white to yellowish lesion measuring $7.5 \times 4.5 \times 3 \mathrm{~cm}$. Cut surface of the lesion showed grey white firm areas with intervening yellowish fatty areas (Figure 1). The epididymis showed a cyst measuring $0.4 \mathrm{~cm}$ filled with serous fluid. The spermatic cord was unremarkable.

Case 2: A 28 yr old lady presented with long standing painless swelling in right gluteal region. Clinical diagnosis of lipoma was made. Local examination showed single well defined mass in right gluteal region, mobile and non tender. Skin over the swelling was normal. On general examination, no clinical signs of tuberous sclerosis were seen. Systemic examination did not reveal any other significant clinical findings. The mass was excised in toto and sent for histopathological examination. Grossly there was a fibrofatty mass measuring $11.5 \times 8 \times 7 \mathrm{~cm}$ covered by elliptical skin measuring $10.5 \times 4.5 \mathrm{~cm}$. Cut surface showed predominantly fatty areas with intervening grey white firm areas and dilated and congested vessels in few areas (Figure 2). 
Histopathological examination of both cases showed a benign lesion comprising of admixutre of lobules of mature adipose tissue with conglomerate of thick walled blood vessels and smooth muscle bundles (Figure 1 \&Figure 2). The paratesticular lesion, in addition, showed admixture of mixed inflammatory infiltrate, infarction and fat necrosis. Testis and epididymis showed features suggestive of subacute epididymo-orchitis (Figure 1). Epididymis also showed a tiny benign cyst lined by single layer of flattened epithelium. The spermatic cord structures including vas, were unremarkable. On immunohistochemistry the smooth muscle cells were positive for Vimentin, Smooth muscle actin(SMA) and Desmin (Figure 3). HMB-45 was negative in both cases. Special stain for elastin showed reduced elastin content with distorted internal elastic lamina in the thick walled vessels (Figure 3). Both the cases were reported as Extrarenal angiomyolipoma.

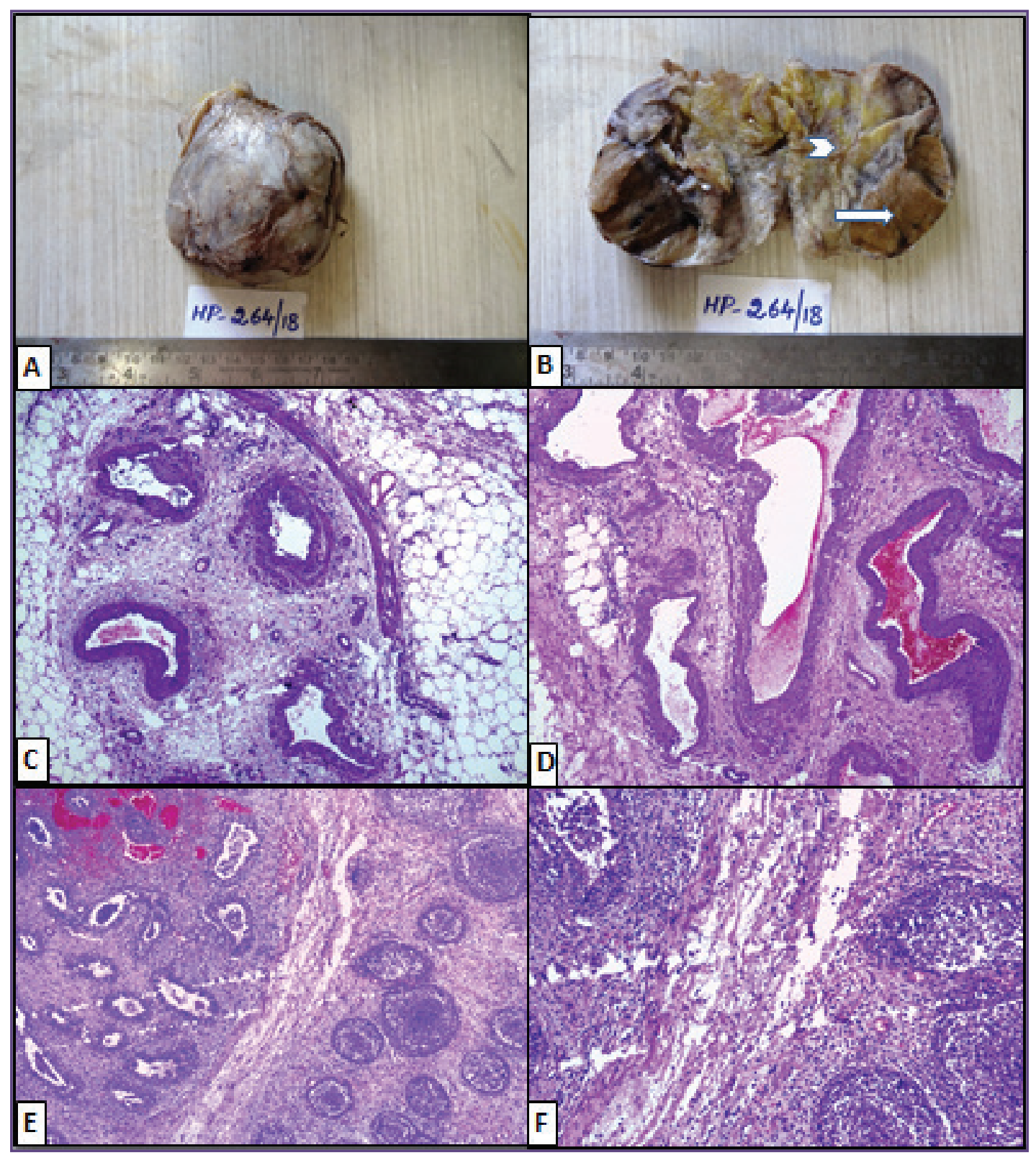

Fig. 1: A \& B: Right orchidectomy specimen (case 1) showing testis (arrow) with a paratesticular lesion showing grey white and fatty areas (arrow head), C \& D: Microscopy showing admixture of thick walled blood vessels with adipose tissue and smooth muscle bundles. E \& F: Microscopy showing associated epididymo orchitis with subacute inflammatory infiltrate in the testis and epididymis. H \& E stain, 100X. 


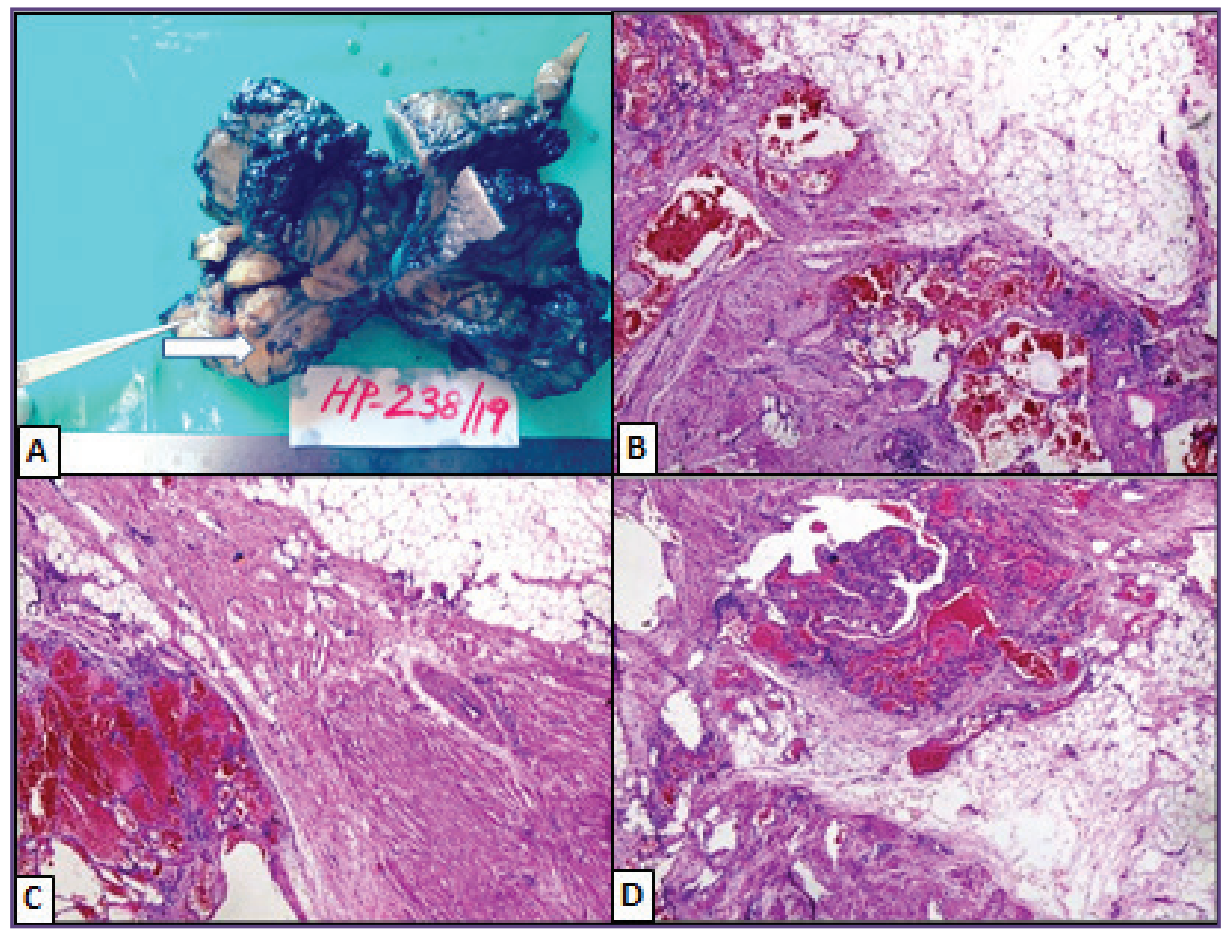

Fig. 2: A: Excised gluteal swelling (Case 2) showing skin covered fibrofatty mass showing fat lobules with focal collection of dilated and congested blood vessels (arrow), B,C,D: showing admixture of congested blood vessels with adipose tissue and smooth muscle bundles. H \& E stain, 100X.

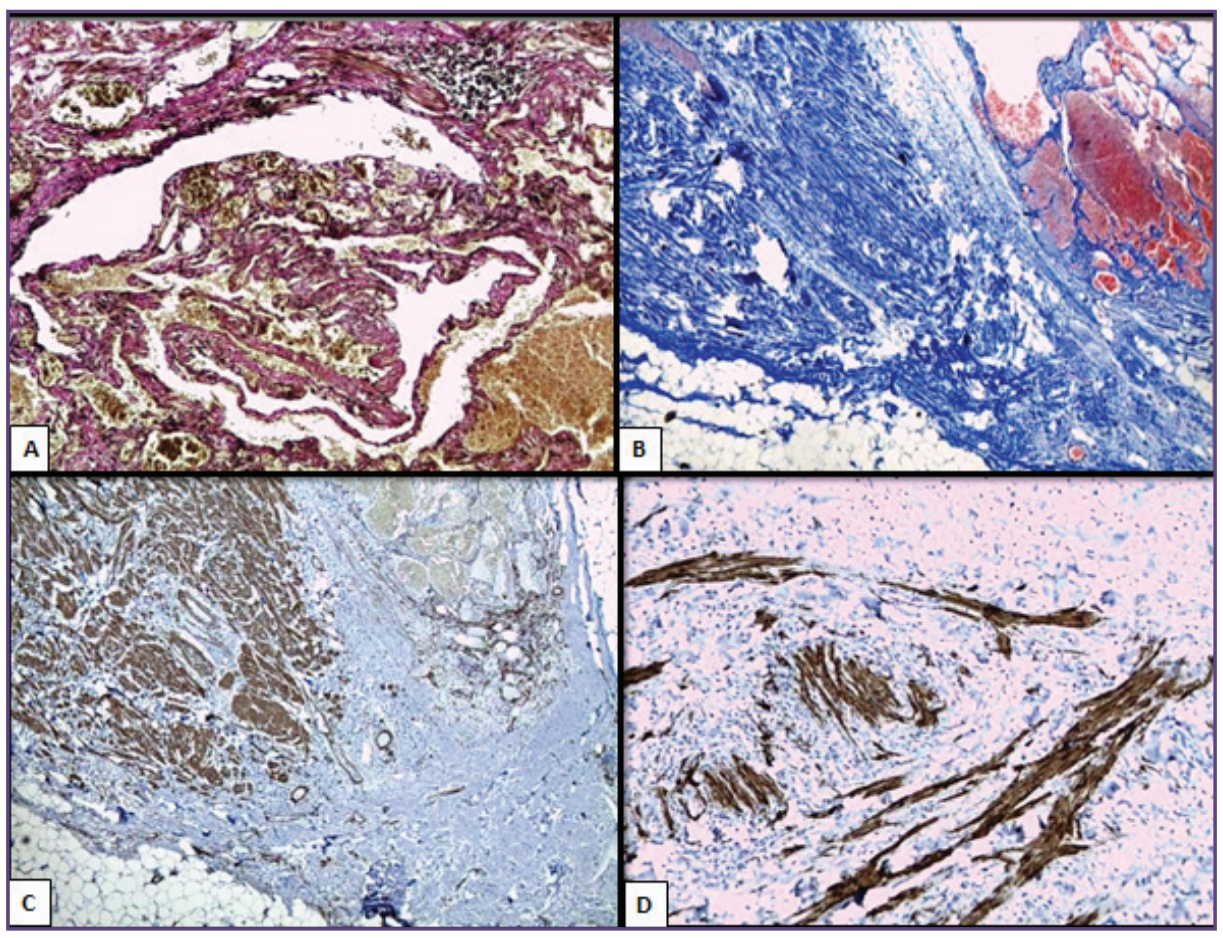

Fig. 3: Elastic stain(A) showing blood vessels with reduced and disrupted elastic lamina. Masson's trichrome stain (B) showing increased fibrosis surrounding the blood vessels. H \& E stain, 100x. C \& D: Immunohistochemistry showing smooth muscle bundles positive for SMA (C) and Desmin (D). 100X. 


\section{Discussion}

Angiomyolipoma was originally described by Fischer in 1911 and it was defined by Morgan in 1951. It is a rare tumor with an incidence of $0.13 \%$ to $0.3 \%$ in the general population. ${ }^{[1]}$ Renal AMLs account for $1 \%$ of renal lesions, occurring more commonly in women. ${ }^{[5]}$ Extrarenal angiomyolipomas are rare which usually present as incidental lesions during imaging for other conditions. ${ }^{[5]}$ Most common extra renal sites are liver and retroperitoneum. Other sites described include nasal cavity, oral cavity, colon, lung, skin, adrenal glands and bladder. ${ }^{[5]}$

Histologically Angiomyolipomas are composed of mature fat cells, vascular tissue, and smooth muscle. The fatty component appears normal. The vascular component shows tortuous and thick walled vessels, have a lower elastin content than normal vessels, and are commonly surrounded by a cuff of smooth muscle cells. The absence of elastic tissue in the vessels of tumors predisposes the patient to the formation of an aneurysm and spontaneous hemorrhage. ${ }^{[6]}$ PEC tumors show immunohistochemical coexpression of myogenic (smooth muscle actin) and melanocytic (HMB-45, melan A, microphthalmia transcription factor) markers. ${ }^{[7,8]}$ Other markers that are commonly positive in angiomyolipomas include CD34, and CD-68. ${ }^{[6]}$ Our both cases showed the classic triphasic morphology showing admixture of mature fat, blood vessels and smooth muscle bundles. There were no clinical signs of TSC or TSC associated lesions in the first case. In the second case, there were no clinical signs of TSC, however radiological evaluation for TSC associated lesions could not be done as the patient was lost for follow up. First case showed superadded epididymo orchitis with which the patient presented to our hospital. HMB45 immunostain was negative in both cases. Although AML $\mathrm{s}$ are characterized by consistent coexpression of melanocytic and smooth muscle markers, some reports suggest that the expression of HMB-45 may be negative in some extrarenal AMLs. The authors in these reports suggest that the HMB-45 negativity may be explained by the rarity of the epithelioid cells in these cases, and the HMB-45 positivity is often weaker or absent in spindle cells. ${ }^{[9]}$ Our cases also showed paucity of epithelioid cells. The perivascular areas showed predominantly spindle cells which expressed smooth muscle markers. Ceifo et $\mathrm{a}^{[10]}$ reported a case of testicular AML with negative HMB-45. Lin et a ${ }^{[9]}$ described a case of orbital AML with negative HMB-45. It has been documented that ultrasound scan and CT scan can diagnose correctly AMLs of the kidney in $86 \%$ of cases. Nevertheless, ERAMLs are more difficult to diagnose on imaging in view of the fact that they tend to lack fat densities. ${ }^{[11]}$
In 1982, Friis and Hjortrup reported the first ERAML in a 22-year-old female who presented with abdominal pain and weight gain. On exploratory laparotomy, the patient was found to have a $11 \mathrm{~kg}$ retroperitoneal AML. ${ }^{[12]}$ Ditonno et $\mathrm{al}^{[13]}$ have reported the largest series of ERAMLs, involving 40 cases. In their report, the liver was the most common extrarenal location followed by the uterus, retroperitoneum , head, vagina, penis, nasal cavity, hard palate, abdominal wall, fallopian tube, spermatic cord, and colon. Although the majority of ERAMLs are benign, few cases of metastatic and recurrent ERAMLs have been reported. Gupta et al. described two cases of retroperitoneal AML which metastasized to the liver and mediastinum after following surgical resection. Although malignant transformation is difficult to predict, high mitotic activity within the primary tumor was a common factor in both metastatic cases. ${ }^{[14]}$ Although initially thought of as hamartomas, AMLs and related tumors have more recently been proved to be clonal neoplasms. Involvement of the tuberous sclerosis complex 2 (TSC2) which is tumor suppressor gene located on chromosome band 16p13 most accurately characterizes underlying genetic change in AML. Allelic losses at 16 p13 have been found in both sporadic AMLs and those associated with $\mathrm{TSC}^{(7)}$. Regarding prognosis, the classic AML is a benign lesion and surgical excision is curative. However, the epithelioid variant has aggressive behaviour. ${ }^{[7]}$

\section{Conclusion}

Although rare, ERAMLs are being increasingly described with most common extrarenal sites being liver and retroperitoneum. Paratesticular and subcutaneous angiomyolipomas are extremely rare, hence the cases are reported here. Though ERAMLs are usually not associated with tuberous sclerosis, it is important to screen these patients for TSC associated lesions for early diagnosis.

\section{Acknowledgements}

We sincerely acknowledge our technical staff, Mrs P Shivashankari, Mr R Bhuvaneshwaran, Mrs Lavanya Latha, Mrs J Rukmani, for their technical help.

\section{References}

1. Flores-Murcioa FJ, Avila-Bozab MP, Aguirre-Quezadac DE. Scrotal angiomyolipoma: a clinical case. Rev Mex Urol 2013;73:143-5.

2. Yapanoglu T, Yilmaz AH, Ziypak T, Adanur S, Demirci E, Bilen H. Extrarenal Retroperitoneal Angiomyolipoma: A Rare Case. J Urol Surg 2017;4:134-6.

3. Vos N, Oyen R. Renal Angiomyolipoma: The Good, the Bad, and the Ugly. Journal of the Belgian Society of Radiology. 2018; 102: 41, pp. 1-9.

4. Thakur B, Kishore S, Bhardwaj A, Kudesia S. Extrarenal angiomyolipoma in an elderly male: A case to remember. Int J Med Public Health 2015;5:121-2. 
5. Lokhande R, Goyal N, Bhargava S, Patni A. Retroperitoneal Angiomyolipoma: Rare Entity Asian Pac. J. Health Sci 2017; 4:15-18.

6. Hayashi Y, Shiyanagi S, Nagae I, Kasuya K, Katsumata $\mathrm{K}$, Yamataka A et al. Angiomyolipoma of the tunica dartos of the scrotum in infancy. J Ped Surg Case Reports 2015;3:334-7.

7. Sultan G, Masood B, Qureshi H, Mubarak M. Angiomyolipoma of the scrotum: report of a rarely seen case and review of the literature. Turk J Urol 2017; 43: 223-6. doi: 10.5152/tud.2017.26779.

8. Jayaprakash PG, Mathews S, Azariah MB, Babu G. Pure epitheliod perivascular epitheloid cell tumor (epitheliod angiomyolipoma) of kidney: Case report and literature review. J Cancer Res Ther 2014;10:404-6.

9. Lin CY, Tsai CC, Kau HC, Yu WK, Kao SC, Liu CJ. HMB45 negative angiomyolipoma of the orbit: a case report and review of the literature. BMC Ophthalmology 2016; 16:8. doi: 10.1186/s12886-016-0185-5.

10. Ceifo W, Qadri IA, Malik A. Angiomyolipoma of the Testis: A Case Report. Med Surg Urol 2015;4: 155. doi:10.4172/2168-9857.1000155

11. Venyo AKG. A Review of the Literature on Extrarenal Retroperitoneal Angiomyolipoma. International Journal of Surgical Oncology 2016; 2016: http://dx.doi. org/10.1155/2016/6347136.

12. Friis J. Hjortrup A. Extrarenal angiomyolipoma: diagnosis and management. J Urol. 1982;127:528-9.

13. Ditonno P, Smith RB, Koyle MA, Hannah J and Belldegrun A. Extrarenal angiomyolipomas of the perinephric space. J Urol. 1992;147:447-50.

14. Gupta C, Malani AK, Gupta V, Singh J, Ammar H. Metastatic retroperitoneal epithelioid angiomyolipoma. J Clin Pathol. 2007; 60:428-431.

*Corresponding author:

Dr. B. Rajeshwari, Junior consultant, Department of Histopathology, Apollo speciality hospitals, Vanagaram. Kilayanambakkam Chennai - 600095, INDIA Phone: +91 9159955741

Email: rajeshwari.buttannavar@gmail.com

Financial or other Competing Interests: None. 